\title{
Non destructive transient thermal evaluation of delaminations inside a laminate: a thermal processing technique of thermal images
}

\author{
bY D. MAILLET, S. DIDIERJEAN, A.S. HOULBERT \\ and A. DEGIOVANNI (*)
}

(") Laboratoire d'Energétique et de Mécanique Théorique et Appliquée, URA CNRS 875 ENSEM, 2 avenue de la Forét de Haye, F-54500 Vandoeuvre Les Nancy, France.

\begin{abstract}
Delaminations in laminate composite slabs can be detected and measured using a heat pulse excitation on one side and temperature measurement through infrared thermography on either side of the slab. The delamination is characterized in terms of its depth, thermal contact resistance (thickness) and transverse extent. An identification method of the first two defect parameters is developed and experimentally validated. It uses, as input, the Laplace transforms of a local contrast thermogram and, as output, thickness and depth profiles of the possible delamination.
\end{abstract}

\section{Nomenclature}

\begin{tabular}{|c|c|c|c|}
\hline a & diffusivity & $\alpha$ & square root of the reduced Laplace \\
\hline$c$ & specific heat & & variable $p^{*}\left(=e^{2} p / a\right)$ \\
\hline$e$ & thickness & $\lambda$ & conductivity \\
\hline$e_{1}, e_{2}$ & thickness of the two layers on & $\rho$ & mass density \\
\hline & each side of the defect & $\theta$ & Laplace transform of temperature \\
\hline$M$ & experimental Laplace transform & \multirow{2}{*}{\multicolumn{2}{|c|}{ Subscripts }} \\
\hline & of the contrast $\Delta \mathrm{T}^{*}$ & & \\
\hline$p$ & Laplace variable & & reduced quantity (dimensionless) \\
\hline$Q$ & energy by unit area & $d$ & delamination \\
\hline$f$ & radial direction & $r$ & radial direction \\
\hline$R_{C}$ & contact resistance & 1,2 & relative to each layer 1 or 2 \\
\hline$T$ & temperature $(=0$ at $t=0)$ & $f$ & front side \\
\hline$t$ & time & $r$ & rear side \\
\hline$y^{*}$ & reduced depth of the defect $\left(=\mathrm{e}_{1} / \mathrm{e}\right)$ & 0 & relative to sane slab \\
\hline
\end{tabular}

\section{Introduction}

Thermal non destructive evaluation (NDE) of composite materials is rapidly developing for a few years [1 to 11]. It is mainly due to the technical progresses in the fields of transient infrared thermography and of rapid data acquisition systems for a later processing on a computer. In these techniques, the part to be checked can be stimulated through photothermal excitation, while the temperature field on part of its surface is monitored using infrared radiometry. The presence of a possible defect affects the internal heat diffusion process and therefore modifies the measured surface temperature field, allowing therefore the detection of a defect.

This work is focused on delamination defects, that is air gaps between two plies of the laminate. Let use note that the complete NDE process of a part requires first the detection of a possible anomaly at some location, second the identification of its nature (discrimination) and third the estimation (measurement) of its parameters (size, depth...). The method presented here concerns the third point, even if point one constitutes a direct output. 


\section{Thermal non destructive evaluation method}

\subsection{Defect characterization}

A delamination located in a composite slab of infinite radial ( $r$ ) extent is outlined in figure 1 . This the use of homogen fiber reinforced polymer where the number of plies is big enough to allow and where the perized equivalent axial $(z)$ thermal properties (diffusivity a and conductivity $\lambda$ ) plane with radial (or tran shift of the in-plane fiber orientation makes the material isotropic in this air layer of infinite transverse) properties $a_{r}$ and $\lambda_{r}$. If this delamination $(d)$ is occupied by an compared to the total extent of conductivity $\lambda_{d}$ and of thickness $e_{d}$ (a few micrometers) small resistance $R_{C}\left(=e_{d} / \lambda\right.$ slab thickness $e\left(=e_{1}+e_{2}\right)$, it can be characterized by a contact $\theta 1$, beneath the heated furrounded by two external layers (1 and 2) of sane material. Its depth

\subsection{Thermal contrast}

The previous characterization is valid for a one-dimensional (1D) transient heat transfe created for example by a homogeneous heat pulse on the slab front side, with an absorbed
energy by unit area $Q$.

In that case, if the initial temperature of the slab is uniform $(=0)$ and if heat losses are temperature $T$ veat equation can be solved for each layer. The thermograms, which are the $(z=e)$ of the slab.

If temperature is divided by its asymptotic value ( $Q / \rho c e)$, in order to get rid of the unknown $\left(R_{C} \neq 0\right)$ and a density $Q$, a normalized $T^{\star}$ thermogram can be obtained for a defective slab $\left(R_{C} \neq 0\right)$ and a similar $T_{0}{ }^{*}$ one for a sane slab $\left(R_{C}=0\right)$. These reduced thermograms are plotted the same figures $t^{*}$ ( Fourier number $=a t / e^{2}$ ) in figures $2 a$ (front side) and $2 b$ (rear side). In also plotted. This the difference of the two curves $\Delta T^{*}\left(=T^{\star}-T_{0}^{*}\right)$, the contrast thermogram, is and constitutes its signature.

\subsection{Identification method}

One has shown $[8,9]$ that the Laplace transform $\Delta \theta^{* *}$ of this contrast $\Delta T^{*}$ has the following expression for front (subscript $f$ ) or rear (subscript $r$ ) detection:

$$
\begin{aligned}
& \Delta \theta_{f}^{* *}=\frac{R_{C}^{*} \sinh ^{2}\left[\alpha\left(1-x^{*}\right)\right]}{\sinh (\alpha)\left\{\sinh (\alpha)+R_{C}^{*} \alpha \sinh \left(\alpha x^{*}\right) \sinh \left[\alpha\left(1-x^{*}\right)\right]\right\}} \\
& \Delta \theta_{r}^{* *}=-\frac{R_{C}^{*} \sinh \left(\alpha x^{*}\right) \sinh \left[\alpha\left(1-x^{*}\right)\right]}{\sinh (\alpha)\left\{\sinh (\alpha)+R_{C}^{*} \alpha \sinh \left(\alpha x^{*}\right) \sinh \left[\alpha\left(1-x^{*}\right)\right]\right\}}
\end{aligned}
$$

with : $\quad \Delta \theta^{* *}=\int_{0}^{\infty} \exp \left(-p^{*} t^{*}\right) \Delta T^{*} \mathrm{~d} t^{*} ; R_{C}^{*}=\frac{R_{C}}{e / \lambda} ; x^{*}=\frac{e 1}{e} ; \alpha=\sqrt{p^{*}}$

Equations ( 1 to 3 ) show that contrast $\Delta T^{*}$ is a function of time $t$ (or $t^{*}$ ) and of the two parameters $R_{c}^{*}$ and $x^{*}$ of the defect, while its reduced transform $\Delta \theta^{* *}$ depends on the same parameters and on the reduced Laplace variable $p^{*}$. One very interesting feature of $\Delta \theta^{\star *}$ is that it can be easily calculated, starting from an experimental contrast curve $\Delta T^{*}\left(t^{*}\right.$ or $\left.t\right)$ through 


\section{http://dx.doi.org/10.21611/qirt.1992.032}

numerical time integration - see equation (3). Starting from equations (1) and (2), written for two values $p_{1}^{*}$ and $p_{2}{ }^{*}\left(=4 p_{1}^{*}\right)$ of $p^{*}$, one can show $[8,9]$ :

$$
R_{C}^{*}=\frac{M_{1}^{2}\left[1+\alpha_{2} \sinh \left(\alpha_{2}\right) M_{2}\right] \sinh \left(\alpha_{1}\right) \tanh \left(\alpha_{1}\right)}{\left[1+M_{1} \alpha_{1} \sinh \left(\alpha_{1}\right)\right]\left[-M_{1}+M_{2} \cosh \left(\alpha_{1}\right)-1.5 M_{1} M_{2} \alpha_{1} \sinh \left(\alpha_{2}\right)\right]}
$$

with : $\quad \alpha_{i}=\sqrt{p_{i}^{*}}$ and $\quad M i=\Delta \theta_{r}^{* *}\left(p_{i}^{*}, R_{C^{\prime}}^{*} x^{*}\right)$ for $i=1,2$

using rear-side contrast (exact expression) and :

$$
x^{*}=1-\frac{1}{\alpha_{1}} \ln \left[\left(\frac{M_{2}}{M_{1}}\right)^{1 / 2} \cosh \left(\alpha_{1}\right)+\left(\frac{M_{2}}{M_{1}} \cosh ^{2}\left(\alpha_{1}\right) \cdot 1\right)^{1 / 2}\right]
$$

using front-side contrast (approximate expression, valid for small $R_{C}^{*}$ 's or delaminations close to one of the two faces of the slab) with:

$$
\alpha_{i}=\sqrt{p_{i}^{*}} \quad \text { and } \quad M_{i}=\Delta \theta_{f}^{* *}\left(p_{i}^{*}, R_{C^{*}}^{*}, x^{*}\right) \text { for } i=1,2
$$

\section{Experimental results}

\subsection{Measurement technique}

A test slab $(360 \times 200 \mathrm{~mm}, 2 \mathrm{~mm}$ thick) made out of T300 Carbon epoxy laminate (14 plies) containing artificial delaminations made of disk-shape Teflon films of various thicknesses, depths and radii (manufactured by the Dassault Company) has been used to validate this NDE technique. Heat pulse excitation was produced by a square assembly of four flash tubes located on the focal lines of four cylindro-parabolic reflectors (MECIR stimulation system). The temperature field on either the front or rear side of the test slab was recorded by a 782 SW AGA infrared scanner and acquisition and data storage was done on a DATAMIN board and software plugged into a $386 \mathrm{PC}$ computer.

In real experimental conditions a sane reference slab is seldom available and the reference $T_{O}(t)$ thermogram can be measured, during a unique experiment, on locations where the temperature is uniformly hot (rear side) or cold (front side) on the monitored frames.

Normalization is also done on a local basis, each $T$ or $T_{0}$ thermogram being divided by its local final value - maximum in rear side and minimum in front side as shown on figures 2 . The result of these normalizations is a contrast $\Delta T^{*}$ that does not depend on the non-uniformity of both emissivity and absorbed energy $Q$. Experimental Laplace transforms of the contrast, $M_{1}$ and $M_{2}$-see first equation (3) - can be calculated and reduced resistances $R_{c}^{*}$ (rear side) or reduced depths $x^{*}$ can be calculated on a point basis.

\subsection{Experimental results}

The thermal frames of two flash experiments corresponding to rear - colour illustration $A^{*}$ and front side detection - colour illustration $B$ - have been processed using the above described technique.

A line BB' passing through the centers of defects B4 to B1 (nominal $R_{C}=2.10^{-4} \mathrm{~m}^{2} . \mathrm{K} . \mathrm{W}^{-1}$; nominal $x^{*}=0.36,0.5,0.71$ and 0.86 for front side detection ; radius : $10 \mathrm{~mm}$ ) has been studied and the identified $R_{C}$ and $x^{*}$ profiles are plotted on figures 3 and 4 . 
For rear side detection, one can notice that the identified $R_{c}$ 's (corresponding to equivalent air thickness) are about two times higher than the nominal ones (except for defect B2 where there is a 6 factor). These over-resistances may stem from air delaminations between the two $25 \mu \mathrm{m}$ teflon sheets of the artificial defect (hypothesis confirmed by an ultrasonic image).

For front side detection, the agreement between nominal and identified depths is better, differences staying always lower than $6 \%$ (of the slab thickness) even if edge effects can affect the defect B4's identified depth.

In another rear side detection experiment, one of the four flash tubes could not operate properly (no heat pulse production for this tube). Figure 5 a shows the absolute contrast profile $\Delta T\left(=T-T_{0}\right)$ for the same line of $B$ defects as before at $2.16 \mathrm{~s}$ after the pulse : it is very much affected by radial heat transfer since its base line is tilted with respect to the horizontal.

In figure $5 b$ the locally normalized contrast $\Delta T^{*}$ profile is plotted for the same experiment : the previous tilt in the base line is strongly reduced. The corresponding $R_{C}$ profile is shown in figure $5 c$ : it is very much consistent with the one obtained from the first experiment - see figure 3 - in spite of the highly non homogeneous front side excitation.

\section{Conclusion}

These results yielded by the thermal NDE technique and by data processing through contrast normalization on a local base followed by Laplace transformation, seem to be very promising. One feature of this quantitative infrared method is that it completely departs from classical thermal image processing techniques and rather constitutes a thermal processing of transient intensity fields. Its main advantages can be quoted:

- an important area can be checked in one experiment, even if heat absorption is not perfectly uniform;

- no surface treatment or painting of the part to be checked is necessary (it is a truly non contact technique by opposition to ultrasonic NDE);

- an integral technique is used that reduces the measurement noise (Laplace transform filtering);

- detection is straightforward and delamination diameters can be estimated using contact resistance profiles;

- production of delamination depth or thickness profiles can be automated and the same is true for the creation of depth or tickness images.

\section{REFERENCES}

[1] VAVILOV (V.P.) and TAYLOR (R.). - Research Techniques in NDT, Ed. 5, Academic Press, London, 1982.

[2] BALAGEAS (D.), KRAPEZ (J.C) and CIELO (P.). - Pulsed photothermal modeling of layered materials. J. Appl. Phys., 59, 1986, 348-357.

[3] BOSCHER (D.M.), DEOM (A.A.), GARDETTE (G.) and BALAGEAS (D.). - Thermal NDT of carbon epoxy laminates. Proc. Eurotherm 4, Nancy, June 28-29, 1988, 112-115.

[4] CIELO (P.), MALDAGUE (X.), DEOM (A.A.) and LEWARK (R.). - Thermographic non destructive evaluation of industrial materials and structures. Material Evaluation, 45,1987, 452-466.

[5] LAMINE (A.S.), MAILLET (D.) et DEGIOVANNI (A.). - Modélisation et détection d'un défaut limité dans un matériau composite. Proc. Eurotherm 4, Nancy, June 28-29, 1988, 101-104.

[6] LAMINE (A.S). - Caractérisation de defauts dans les matériaux composites par thermographie infrarouge. Thesis, INPL Nancy, nov. 1988.

[7] HOULBERT (A.S.), LAMINE (A.S) et DEGIOVANNI (A.). - Modélisation d'un défaut limité en vue du contrôle non destructif des multimatériaux isotropes. Int. J. Heat Mass Transfer, $34,4 / 5,1991,1125-1138$.

[8] MAILLET (D.), HOULBERT (A.S.), DIDIERJEAN (S.) and DEGIOVANNI (A.). Identification of a contact resistance located at any depth inside a laminated composite material using experimental Laplace transforms and thermography. Proc. $4^{\text {th }}$ Annual Inverse Problems in Engineering Seminar, East lansing, Michigan, June 10-11, 1991.

[9] MAILLET (D.). - Apport des méthodes analytiques à l'identification des paramètres et à la conduction inverse en thermique. Thèse d'Etat, INPL Nancy, dec.20, 1991. 
[10] VAVILOV (V.P.), DEGIOVANNI (A.), DIDIERJEAN (S.), MAILLET (D.),SENGOULIER (A.A.) and HOULBERT (A.S.) - Thermal flaw detection and tomography of carbon fiberreinforced articles. The Soviet Journal of Non Destructive Testing, 609-619, Plenum Pub. Corp., 1992, translated from Defektoskopiya, 9, 3-14, sept. 1991.

[11] MAILLET (D.), HOULBERT (A.S), DIDIERJEAN (S.), LAMINE (A.S.) and DEGIOVANNI(A.). - Non destructive thermal evaluation at LEMTA-Delaminations inside a laminate : the identification problem, Article ref.58/59-A92-431, to be published in Composites Science and Technology.

Q $\left(J / m^{2}\right)$

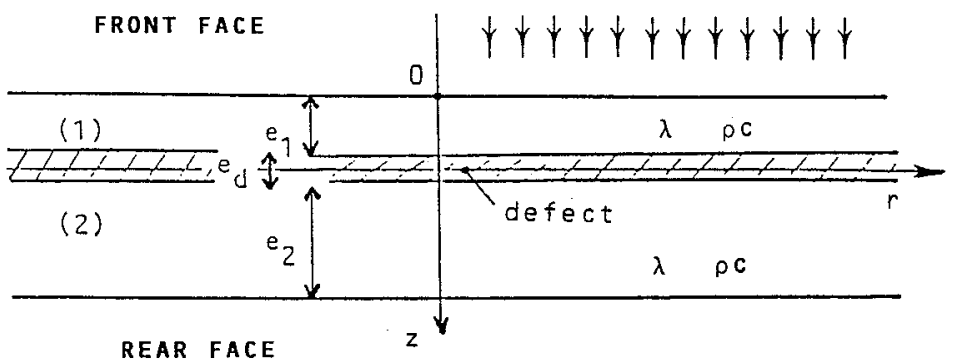

Fig. 1. - Geometry of the one-dimension model
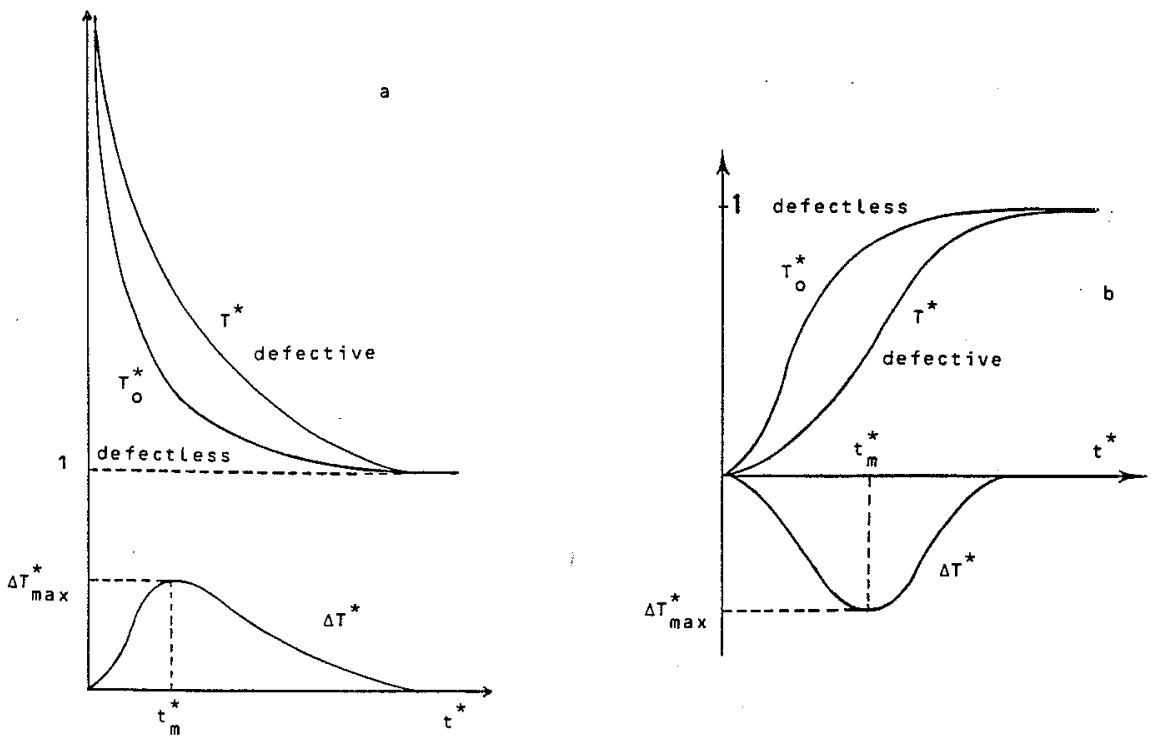

Fig. 2. - Thermograms and contrasts

a - front side detection

b - rear side detection 
http://dx.doi.org/10.21611/qirt.1992.032
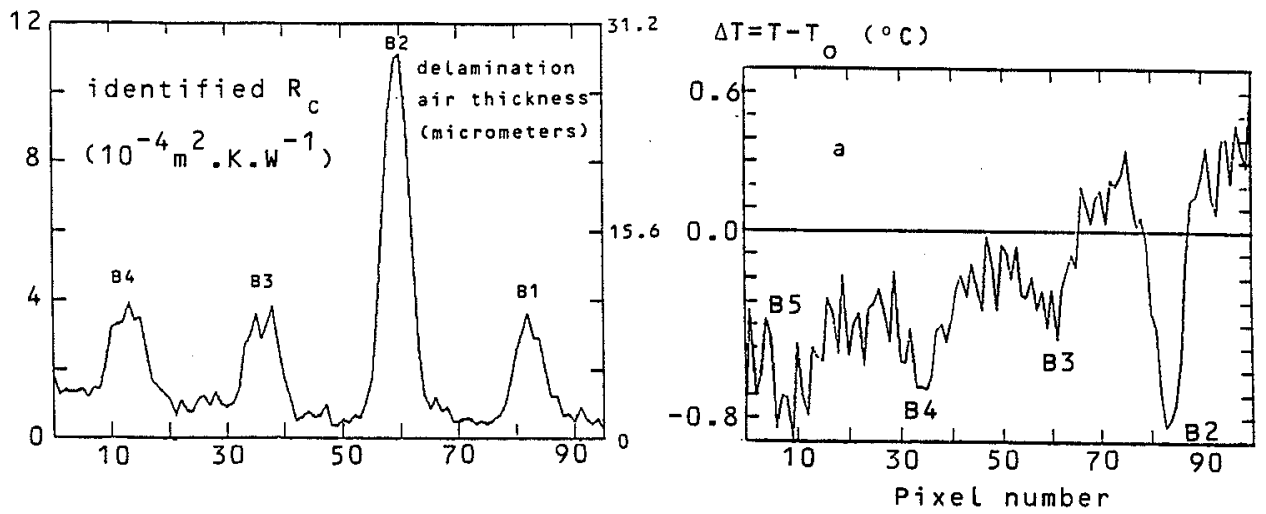

Fig. 3.- Identifled contact resistance $R_{C}$ B-B' profile
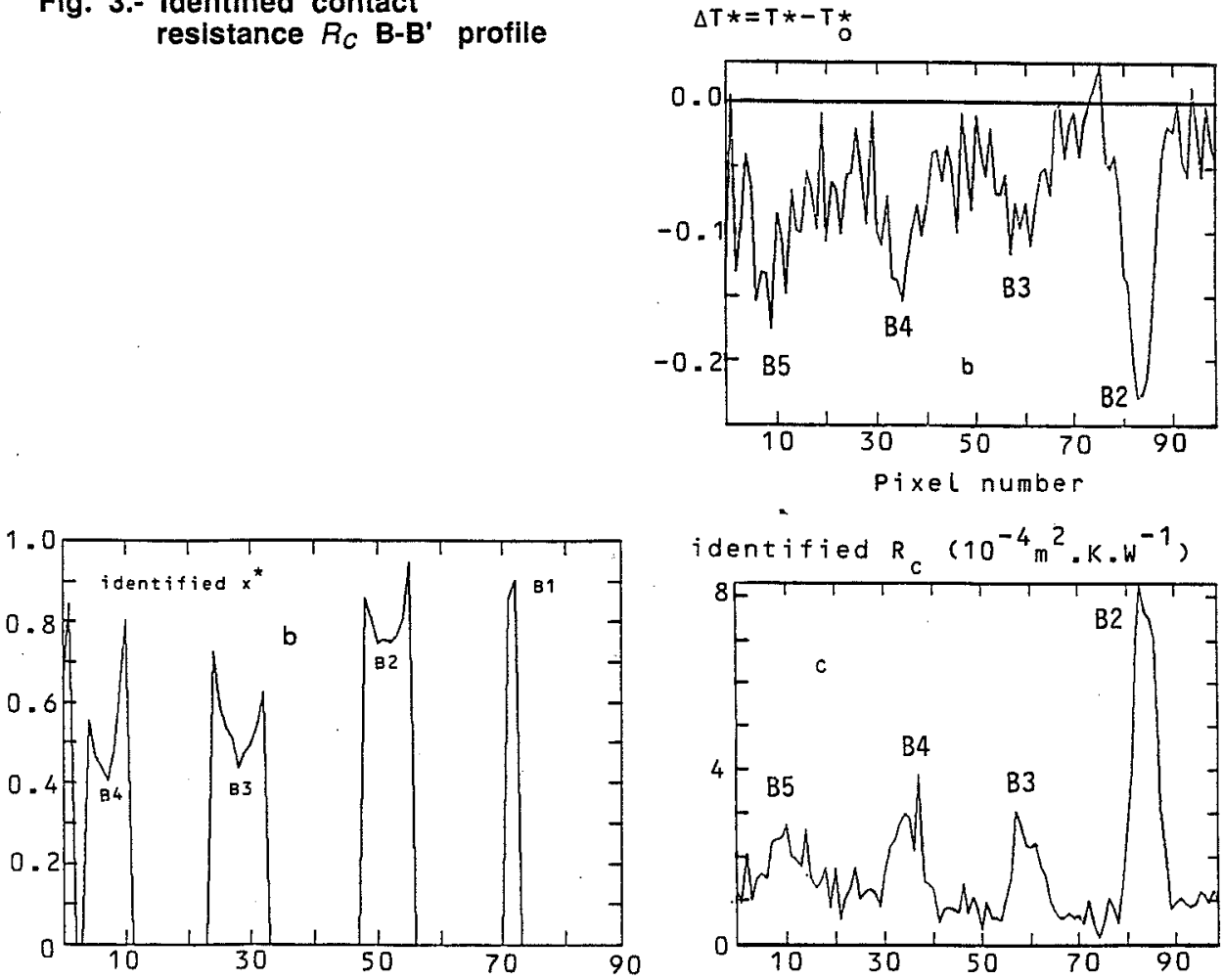

Fig. 4. - Identified reduced depth $x^{*}$ (defect depth beneath front side divided by slab thickness) B-B' profile

Fig. 5. - Rear side experiments with defectuous stimulation $B$ defects

a - absolute contrast $\Delta T$ profile $(t=2.16 \mathrm{~s})$

b - contrast $\Delta T^{*}$ profile $(t=2.16 \mathrm{~s})$

$c$ - identified resistance $R_{C}$ profile 